\title{
Improving the Power System safety in Hospitals by means of periodical SFRA tests on Medical Isolation Transformers

\author{
G. Bucci ${ }^{1}$, F. Ciancetta ${ }^{1}$, A. Fioravanti ${ }^{1 *}$, E. Fiorucci ${ }^{1}$, and A. Prudenzi ${ }^{1}$
} \\ ${ }^{1}$ Department of Industrial Engineering Information and Economics, University of L'Aquila \\ Via G. Gronchi 18, 67100 L'Aquila (Italy) \\ Phone/Fax number: +39 0862 434838/434803, e-mail*: andrea.fioravanti@ graduate.univaq.it
}

\begin{abstract}
The paper has the main aim of demonstrating a possible use of the well consolidated monitoring SFRA (Sweep Frequency Response Analysis) technique for preventive maintenance procedure applied to isolation medical transformers operating in hospitals' group 2 medical locations. In particular, a novel testing platform has been implemented that can perform the monitoring test on IT$M$ during normal periodical verification provided by specific standard.

In the paper, some results of the practical application of such a platform to several transformers in currently in operation in various Italian hospitals have been reported.
\end{abstract}

Keywords. SFRA, Isolation transformers, Medical equipment, Resilience.

\section{Introduction}

IT-M medical transformers are transformers designed to isolate patients and hospital operators from electric shocks and to protect the equipment from power surges or faulty components [1]. They are used to provide a safer environment by minimizing the potential hazards caused by contact voltages and avoiding power loss even in single fault conditions.

It is well known that a SFRA test can be applied to transformers in order to assess winding conditions without the need to open the transformer for inspection [2]. The test is capable of identifying either core or windings movement, winding short circuit, junctions loosening and any other internal fault [3].

Therefore, the SFRA technique could help hospital maintenance personnel identify tempestively even slightly damaged isolation transformers, allowing to replace the faulted IT-M before severe failure and subsequent power outage occur [4].

\section{SFRA technique}

Most passive electrical equipment, such as in the case of the isolation transformer, can be considered RLC circuits and at the scanning frequencies produce a unique signature [5]. Summing up, in a circuit powered with an alternating signal with frequency $\mathrm{f}$, and angular frequency $\boldsymbol{\omega}=\mathbf{2} \boldsymbol{\pi} \boldsymbol{f}$ we can identify discrete components such as:

- resistance R: invariable with frequency;

- capacitive reactance $X_{c}=1 / 2 \pi f C$ which reduces with increasing frequency;

- inductive reactance $X_{c}=\mathbf{2 \pi} \boldsymbol{f} \boldsymbol{L}$ which increases with increasing frequency.

The impedance of individual components varies with frequency, as shown in Figure 1.

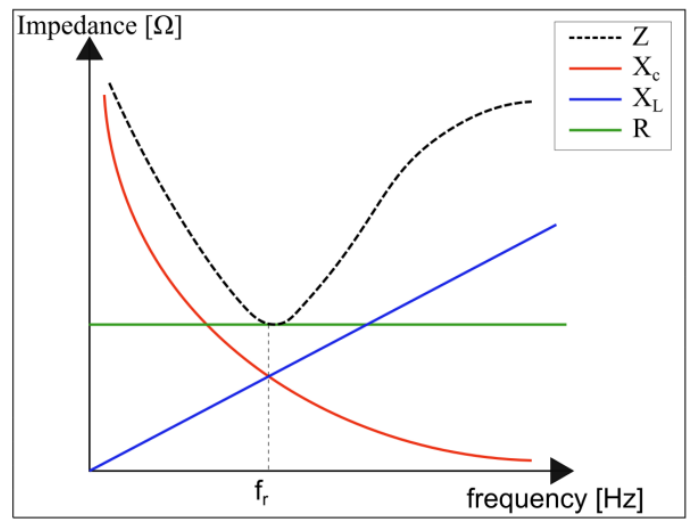

Fig. 2. Impedance curves of electrical components

The transformer is considered a complex network of RLC components connected to each other and which may also have more resonance frequencies. Any mechanical and electrical variation can cause a change in the parameters which affects a change in the frequency response.

The SFRA test is performed by injecting a low voltage sinusoidal signal at one end of the transformer winding and measuring the voltage at the other end of the transformer winding itself. The ratio between the input and output voltage is then displayed in the frequency domain in $\mathrm{dB}$. The impedance of the transformer under test attenuates and breaks the injected signal [6].

To remove the effects of the test leads, a three-conductor system is used, one to inject the signal at point A (input), one to measure the voltage at point $\mathrm{A}$ and the third to simultaneously measure the voltage at point B (output). The measurement circuit is shown in Figure 2.

The ratio between the voltages measured in $\mathrm{dB}$ is: 


$$
T F=20 \log _{10} \frac{V_{2}}{V_{1}}
$$

Experience has shown that the response spectrum can be divided into different sub-bands, with undefined boundaries and even overlapping areas, more sensitive to different types of faults [4].

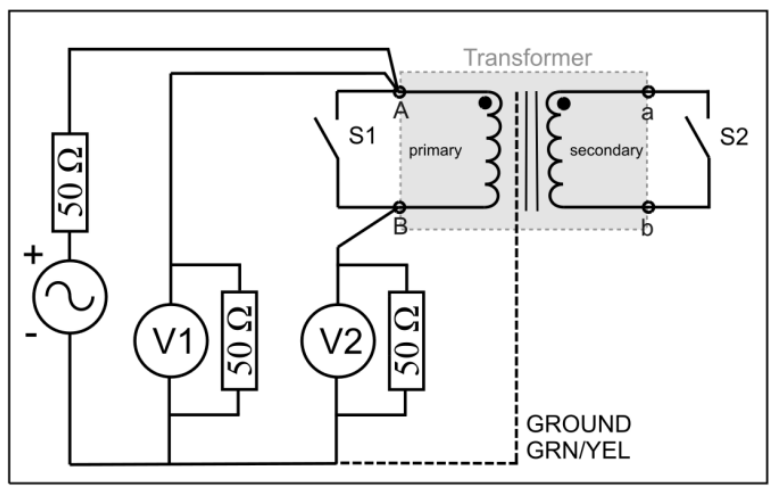

Fig. 2. Circuit connection for SFRA test

The SFRA test is a comparative test where the TF obtained on the new or newly assembled transformer is compared with the TF after the failure or after a certain time period [7].

The variations between the two responses at low frequencies, where the inductive part of the circuit is prevalent, derive from the deformation of the magnetic core or from short-circuits while the variations at the medium frequency depend on the windings since the capacitive part is preponderant with respect to the inductive part. Variations at high frequencies can be cut as they depend on the connection of the measuring instrument and the placement of the cables.

\section{SFRA platform}

To carry out the tests in a repetitive way, we have developed a low-cost SFRA test system that is light and easy to transport, designed to control transformers installed in a hospital environment $[8,9]$.

The hardware platform can be easily connected to the Notebook, via USB port, where the program for starting the test, displaying tables and graphs and managing the results can be installed.

The program was created in the NI Labview 2018 environment and then, the executable application was created for all Windows PCs through the LabVIEW Application Builder,

Inside the electronics enclosure, the electronic circuit depicted in fig. 2 is realized with an Analog Discovery 2 of the Digilent brand connected to the Digilent Discovery BNC.

From the generator of arbitrary functions $( \pm 5 \mathrm{~V}, 14$ bit, 100 Msample / s, bandwidth of $20 \mathrm{MHz}$ ) the coaxial cable that goes to feed the test circuit has been connected.

The two oscilloscope channels $(1 \mathrm{M} \Omega, \pm 25 \mathrm{~V}$, differential, 14 bit, 100 Msample / s, bandwidth $30 \mathrm{MHz}$ were placed in parallel with two $50 \mathrm{ohm} \mathrm{BNC} \mathrm{resistors}$ and were connected to the two coaxial cables which they pick up the signals injected at the beginning and at the end of the winding of the transformer.

All the masses of the coaxial circuits and cables are then connected to the terminal which must be connected in the test phase to the transformer screen and therefore to the metal masses of the transformer.

This circuit, despite the low cost, has managed to exceed the standard requirements imposed by the IEC60076-18 standards for the SFRA measurement system:

- accuracy of the Vout / Vin ratio better than \pm 1 $\mathrm{dB}$ in the range from $+10 \mathrm{~dB}$ to $-80 \mathrm{~dB}$;

- accuracy of the Vout / Vin ratio better than \pm $0.3 \mathrm{~dB}$ in the range from $+10 \mathrm{~dB}$ to $-40 \mathrm{~dB}$;

- best phase measurement accuracy of $\pm 1^{\circ}$ in the range from $+10 \mathrm{~dB}$ to $-40 \mathrm{~dB}$;

- maximum bandwidth of $10 \mathrm{~Hz}$ for frequencies below $100 \mathrm{~Hz}$ and less than $10 \%$ of the measured frequency above $100 \mathrm{~Hz}$;

- $\quad$ input voltage up to $50 \mathrm{Vpp}$;

- $50 \Omega$ characteristic impedance and attenuation of less than $0.3 \mathrm{~dB}$ at $2 \mathrm{MHz}$ for coaxial cable input and output;

- internal impedance of the generator, impedance of the input measuring instrument and characteristic impedance of the cable of 50 $\Omega \pm 2 \%$ for the entire measurement range.

\section{SFRA test}

The most common fault on the transformer is the mechanical deformation due to bad transport or to accidental causes during assembly and coil-coil or shield-coil faults due to forces caused by short-circuit conditions. The measures should therefore be made as well as periodically, even after one of these episodes $[10,11]$.

Other faults that are difficult to perceive as slow tightening could reduce the operating capacity of the transformer and therefore of the operating room.

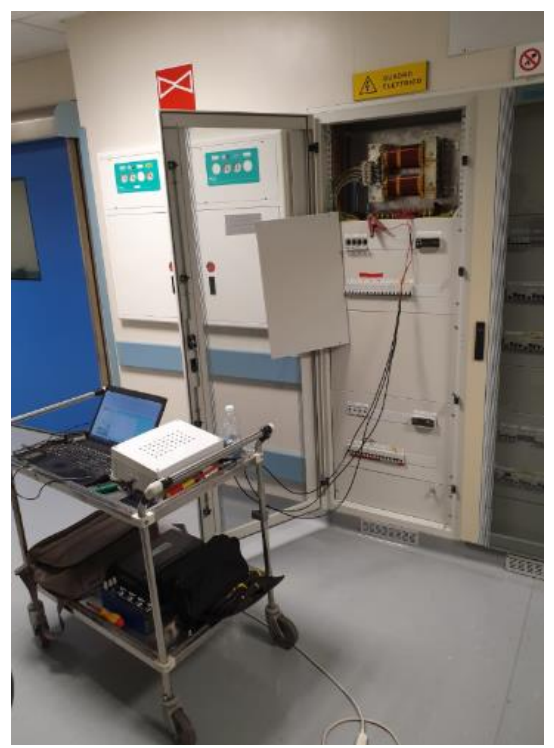

Fig. 3. SFRA test on isolation medical transformers of the hospital group 2 medical location 
During the stops of the operating rooms of the hospital for sanitization and maintenance of the same, we have tested our platform on the isolating transformers as shown in figure 3.

In the first test phase, the 10kVA Thytronic medical isolation transformer that fed the eyes operating room was analyzed by SFRA technique. The transformer was measured by placing the terminals on the primary circuit and leaving the secondary open (see fig. 4-7).

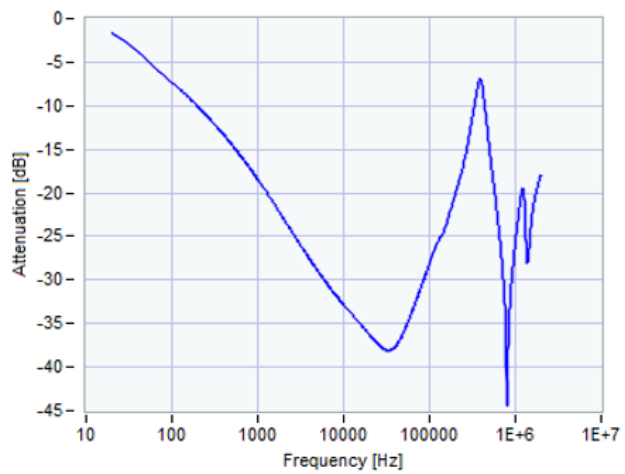

Fig. 4 Open circuit SFRA test on the eye operating room transformer: amplitude response graph

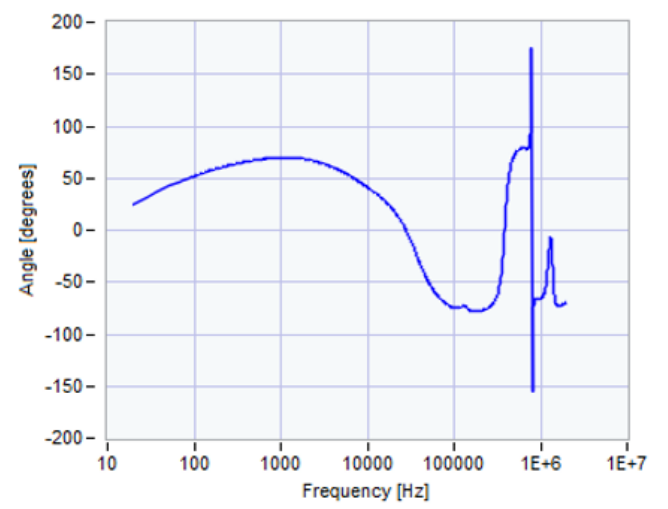

Fig. 5 Open circuit SFRA test on the eye operating room transformer: angle response graph

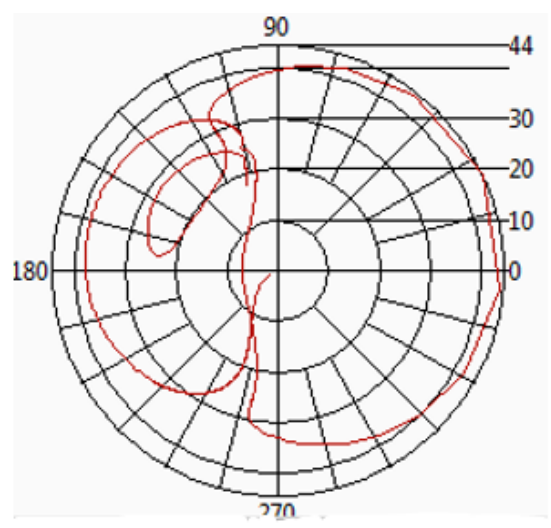

Fig. 6 Open circuit SFRA test on the eye operating room transformer: polar response graph

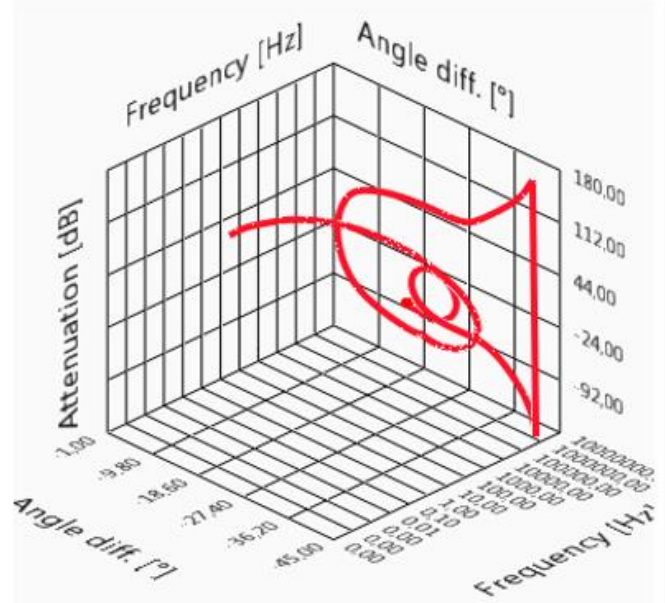

Fig. 7 Open circuit SFRA test on the eye operating room transformer: 3D response graph

In the second test phase, 4 Siemens $4 \mathrm{kVA}$ isolation transformers were analyzed. These transformers fed the group 2 electric sockets of the operating rooms 6 of the $\mathrm{BOE}$ (emergency operating section) and A of the DEA (emergency and acceptance department).

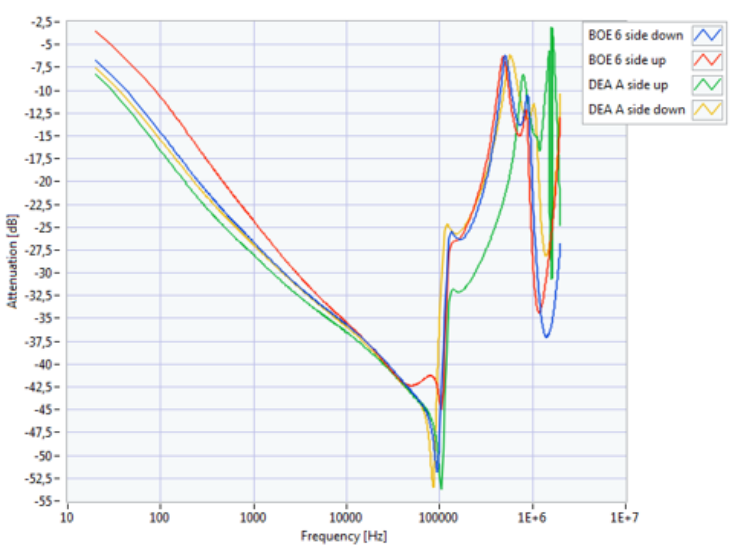

Fig. 8 Open circuit SFRA test on DEA and BOE transformers: amplitude graph

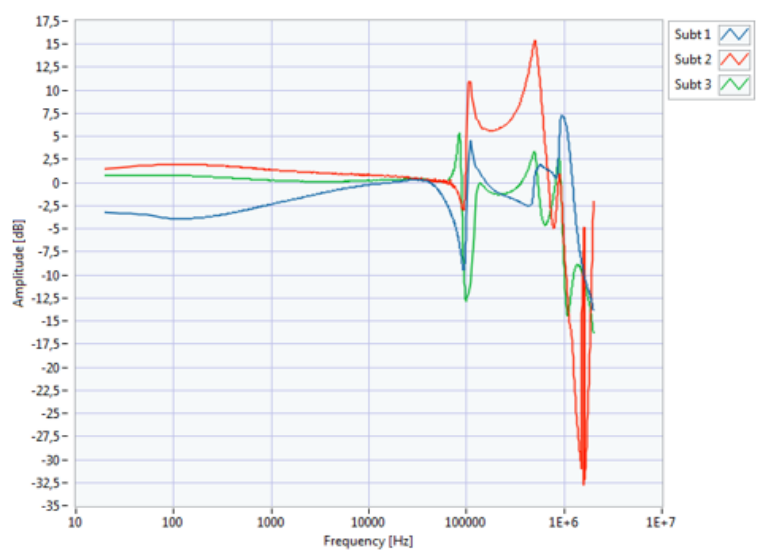

Fig. 9 Amplitude differences in the open circuit test on DEA and $\mathrm{BOE}$ transformers 


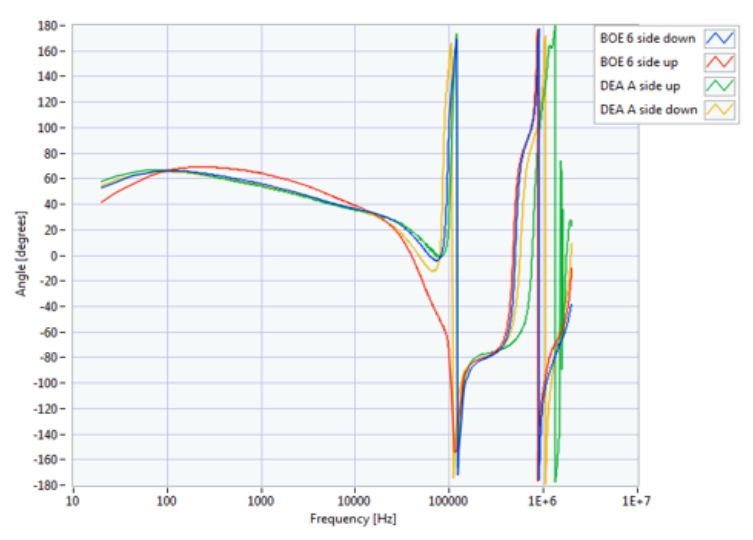

Fig. 10 Open circuit SFRA test on DEA and BOE transformers: angle graph

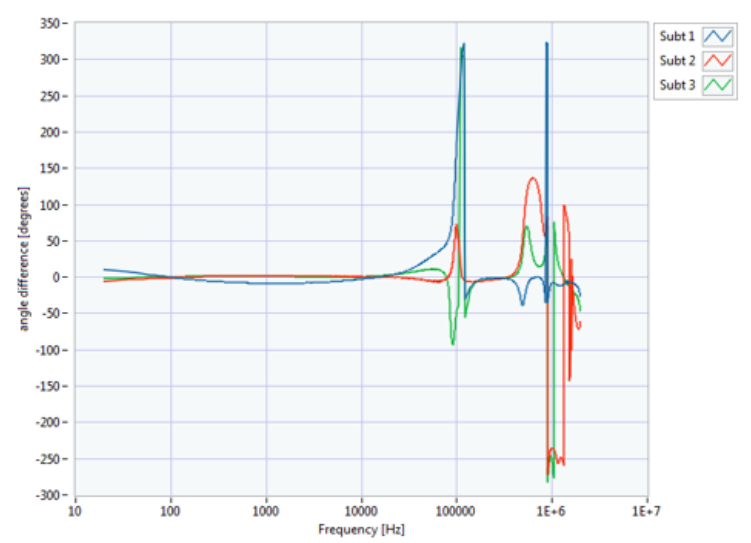

Fig. 11 Angle differences in the open circuit test on DEA and BOE transformers

\section{Conclusions}

Among the negative aspects of the increasingly widespread use of technology in medical practice, there is an increase in dependence on electricity. Failure to operate equipment that is entrusted with vital function for the patient or failure to operate a device during nonrepeatable treatment may increase hospital risk.

The electrical blackout, caused by a transformer failure, is an important, rapidly evolving technological risk that compromises the regular course of work. In the event of failure of the isolation transformer, this risk is accentuated and the interruption of electricity in group 2 environments can also cause serious damage to patients; as these rooms are used in surgical operations, or intracardiac interventions, or where the patient is subjected to vital treatments for which the lack of power supply can be life threatening.

The goal of the work being developed, is to increase the level of safety in hospitals through new techniques to identify the possible occurrence of faults on the isolation transformers.

The Sweep frequency analysis response is one of the diagnostic tools actually useful for detecting possible mechanical and electrical faults that modify the impedance of the transformer.

The platform used in this paper is a powerful means of analyzing a low cost and fast way of the performance of isolation transformers installed within local doctors. Several repetitive measures must be made before commissioning and during normal operation at regular intervals, to detect the fault and to intervene before further effects.

In order for the method to be valid, it is necessary to test both healthy transformers and transformers with known faults to fill a complete database with all the information necessary for detailed analysis.

This document has shown some data that can be obtained by carrying out the SFRA measurement on transformers, actually installed in a hospital environment, of different brands and not.

\section{References}

[1] A. Prudenzi, A. Fioravanti, M. Regoli, "A low-cost IoT solution for power availability improvement in hospitals", International Conference on Renewable Energies and Power Quality (ICREPQ'18), Salamanca (Spain), 21 th to 23th March, 2018.

[2] G. Ramakrishaprabu M. E., P. Prabakaran. B. E., "Diagnosis of transformer using frequency response analysis", International Journal of Engineering Applied Sciences and Technology, 2018,Vol. 3, Issue 2, ISSN No. 2455-2143, Pages 16-20.

[3] Adel El-Faraskoury, "Experiences of Sweep Frequency Response Analyser for the Diagnosis of Transformer Winding Damage", IEEE- MEPCON 2015, Mansoura University, Egypt, December 15-17, 2015, DOI: 10.13140/RG.2.1.2787.0805

[4] G. Bucci, F.Ciancetta, A. Fioravanti, E. Fiorucci, A. Prudenzi, "Application of SFRA for diagnostics on medical isolation transformers", International Journal of Electrical Power \& Energy Systems,Volume 117, May 2020, 105602

[5] G. M. Kennedy, A. J. McGrail and J. A. Lapworth," Transformer sweep frequency response analysis (SFRA)", energize, page 28-33, October 2007.

[6] Mohd Fairouz Bin Mohd Yousof, Thesis:"Frequency Response Analysis for Transformer Winding Condition Monitoring", The University of Queensland,2015

[7] Martin Brandt, Miroslav Gutten, Tomasz Koltunowicz, Pawel Zukowski, "Analysis of winding fault in electric machines by frequency method", 2018 Elektro, Mikulov (Czech Republic), 21-23 May 2018, DOI: 10.1109/ELEKTRO.2018.8398298

[8] A. Prudenzi, A. Fioravanti, M. Regoli, "Smartening hospital electrical distribution for enhancing resilience", 2018 110th AEIT International Annual Conference, Bari (Italy), 3-5 Oct. 2018, DOI: 10.23919/AEIT.2018.8577293

[9] A. Prudenzi, A. Fioravanti, M. Regoli, “A Low-Cost Internet of Things Integration Platform for a Centralized Supervising System of Building Technology Systems in Hospitals", 2018 IEEE International Conference on Environment and Electrical Engineering and 2018 IEEE Industrial and Commercial Power Systems Europe (EEEIC /I\&CPS Europe), Palermo (Italy), 12-15 June 2018, DOI: 10.1109/EEEIC.2018.8494473

[10] Esam Al Murawwi, Braham Barkat, "A new technique for a better sweep frequency response analysis interpretation", 2012 IEEE International Symposium on Electrical Insulation, San Juan, PR, USA, 10-13 June 2012, DOI: 10.1109/ELINSL.2012.6251491

[11] J. C. Gonzales, E. E. Mombello, "Automatic detection of frequency ranges of power transformer 
transfer functions for evaluation by mathematical indicators", 2012 Sixth IEEE/PES Transmission and Distribution: Latin America Conference and Exposition (T\&D-LA), Montevideo (Uruguay), 3-5 Sept. 2012, DOI: 10.1109/TDC-LA.2012.6319080 